\title{
Editorial
}

\section{Structural Dynamical Monitoring and Fault Diagnosis}

\author{
Jiawei Xiang, ${ }^{1}$ Yaguo Lei, ${ }^{2}$ Yanxue Wang, ${ }^{3}$ Yumin He, ${ }^{4}$ Changjun Zheng, ${ }^{5}$ and Haifeng Gao ${ }^{1}$ \\ ${ }^{1}$ College of Mechanical and Electrical Engineering, Wenzhou University, Wenzhou 325035, China \\ ${ }^{2}$ School of Mechanical Engineering, Xian Jiaotong University, Xi'an 710049, China \\ ${ }^{3}$ Department of Mechanical Engineering, Technology University of Darmstadt, 64289 Darmstadt, Germany \\ ${ }^{4}$ Department of Structural Engineering, University of California, San Diego, CA 0085, USA \\ ${ }^{5}$ Department of Civil Engineering, University of Siegen, 57076 Siegen, Germany \\ Correspondence should be addressed to Jiawei Xiang; jwxiang@wzu.edu.cn
}

Received 4 July 2015; Accepted 22 July 2015

Copyright (C) 2015 Jiawei Xiang et al. This is an open access article distributed under the Creative Commons Attribution License, which permits unrestricted use, distribution, and reproduction in any medium, provided the original work is properly cited.

Structural dynamical monitoring and fault diagnosis are of great importance to diagnose/prognoses faults/failures of structures. To detect faults in the components of structures, many novelty methods are proposed $[1,2]$. Lei et al. summarized the methods of the present fault diagnosis of planetary gearboxes, including modeling methods, signal processing methods, intelligent diagnosis methods, and other novelty methods [3].

Although the present methodologies and techniques are feasible to resolve some problems, the emergence of nondestructive tests, structural health monitoring, and equipment evaluation using vibration information demands for the development of numerical simulation and experimental demonstration of new methodologies and techniques. For the numerical simulation of rotor-bearing systems, Xiang et al. presented a class of wavelet-based Rayleigh-Euler rotating beam element using B-spline wavelets on the interval (BSWI) to analyze rotor-bearing system [4]. Further, they proposed a new two-step methodology for detecting multiple faults in structures, in which the singularity detection techniques were employed to decompose the mode shape to reveal singularities and hence the fault locations and the fault severities at the identified locations were then assessed in the second step using the numerical simulation results and the traditional/intelligent optimization techniques $[5,6]$. To detect the faults in real world structures (mechanical systems or civil structures), intelligent classification techniques might be the best way for the practice applications. However, the lack of the faulty samples will lead to the failure to detect faults in real world structural systems. Hence, numerical simulation plays a key role in the present fault detection methodology to obtain the simulated faulty samples to replace the measured ones. Therefore, the objective of this special issue is to present original research and review articles on the latest theoretical developments and experimental techniques in structural dynamical monitoring and fault diagnosis, such as diagnostics/prognostics/machine learning, numerical simulation modeling of structures with faults, structural health monitoring, nondestructive testing and evaluation, equipment evaluation using vibration information, and condition monitoring systems.

In the present special issue, we accepted research articles from Denmark, Japan, China, South Korea, Egypt, Iran, Turkey, Spain, Colombia, and Serbia after a strict peer review process. The research areas were within the topic of the special issue, for example, fault diagnosis of mechanical components (bearings, gears, diesel engine, and shafts), dynamical model updating of mechanical and civil structures, structural health monitoring of civil structures (builds and bridges), fatigue life analysis using vibration signals for the equipment evaluation, the construction of condition monitoring systems using the novel methods for mechanical systems and biomedical systems, lightweight investigation using vibration signals for vehicle engineering, numerical simulation of earthquake waveforms to the condition monitoring of civil engineering structures, and numerical simulation of structures.

For the fault diagnosis of mechanical components, in the paper "Study on Fault Diagnosis of Rolling Bearing Based on 
Time-Frequency Generalized Dimension” by Y. Yuan et al., a novel multifractal fault diagnosis approach using timefrequency domain signals was proposed to detect faults in rolling-element bearings. In the paper "Rotating Machine Fault Diagnosis Based on Optimal Morphological Filter and Local Tangent Space Alignment" by S. Dong et al., the hybrid scheme using particle swarm optimization (PSO) algorithmbased optimal morphological filter and the nonlinear manifold learning algorithm local tangent space alignment (LTSA) was developed to extract the fault features of rolling-element bearings. The paper "Mutual Information-Assisted Wavelet Function Selection for Enhanced Rolling Bearing Fault Diagnosis" by R. Yan et al. improved the performance of the wavelet packet transform (WPT) to classify the faults in rolling-element bearings. In the paper "Fault Diagnosis for Gearbox Based on Improved Empirical Mode Decomposition" by L. Zhao et al., the fault in gears was detected using the orthogonal empirical mode decomposition (OEMD). The paper "An Algorithm of Quantum Restricted Boltzmann Machine Network Based on Quantum Gates and Its Application" by P. Zhang et al. investigated the algorithm of quantum restricted Boltzmann machine network for gear faults identification. The paper "An Adaptive Support Vector Regression Machine for the State Prognosis of Mechanical Systems" by Q. Zhang et al. built up an adaptive support vector regression (ASVR) to predict the faults in descaling pumps. The paper "A Novel Rotor Profile Error Tracing and Compensation Strategy for High Precision Machining of Screw Rotor Based on Trial Cutting of Limited Samples" by Z. Shen et al. presented a trail cutting fault detecting method to trace the rotor profile error and further generate a compensation strategy for the improvement of running precision of screw rotors. The paper "A Method Combining Order Tracking and Fuzzy CMeans for Diesel Engine Fault Detection and Isolation" investigated the hybrid method using order tracking and fuzzy $C$ means for the fault detection of diesel engine. The paper " $\mathrm{A}$ Comparative Study on Multiwavelet Construction Methods and Customized Multiwavelet Library for Mechanical Fault Detection" by J. Yuan et al. discussed the performance of the multiwavelet construction and the corresponding customized multiwavelet library for the application in mechanical fault detection.

For the dynamical model updating of mechanical and civil structures, the paper "Model Updating of Spindle Systems Based on the Identification of Joint Dynamics" by $\mathrm{H}$. Cao et al. presented a method to update the finite element model of spindle. The joint stiffness was identified through the iteration process by minimizing the difference between the simulated frequency response function (FRF) and the measured FRF of the assembly. The paper "A PSO Driven Intelligent Model Updating and Parameter Identification Scheme for Cable-Damper System" by D. Dan et al. proposed particle swarm optimization (PSO) algorithm-based parameters identification method of cable-damper system for the finite element model updating.

For the structural health monitoring of civil structures, in the paper "A Bioinspired Methodology Based on an Artificial Immune System for Damage Detection in Structural Health Monitoring" by M. Anaya et al., the bioinspired methodology using artificial immune system (AIS) is applied to determine faults based on the sudden changes of the ultrasonic signals measured from an aircraft skin panel. The paper "Acoustical Source Tracing Using Independent Component Analysis and Correlation Analysis" by W. Cheng et al. presented a hybrid of independent component analysis (ICA) and correlation analysis scheme to locate the acoustical source of shell structures to indicate the damage locations. The paper "Bridge Damage Severity Quantification Using Multipoint Acceleration Measurement and Artificial Neural Networks" by P. Chun et al. developed a damage severity detection method using accelerators and artificial neural networks. The paper "StayedCable Bridge Damage Detection and Localization Based on Accelerometer Health Monitoring Measurements" by M. R. Kaloop and J. W. Hu developed a structural health monitoring scheme using accelerators, time series analysis, response spectra, and the power density spectra to construct in situ monitor of the damage condition of bridge.

For the fatigue life analysis using vibration signals for the equipment evaluation, the paper "Evolution Balancing of the Small-Sized Wheel Loader Assembly Line" by R. Lai et al. investigated the application of genetic algorithms (GAs) to improve the balancing efficiency and precision of wheel loader assembly line. The paper "Fatigue Life Analysis of Rolling Bearings Based on Quasistatic Modeling” by W. Guo et al. proposed a quasistatic model-based fatigue life analysis method for the rolling-element bearings. Both numerical simulation and experiment investigation were performed to testify the fatigue life prediction model. The paper "NC Machine Tools Fault Diagnosis Based on Kernel PCA and $k$ Nearest Neighbor Using Vibration Signals" by Z. Yuqing et al. proposed a cutting tool processing cycle prediction scheme based on the fault classification using kernel principal components analysis (PCA).

For the construction of condition monitoring systems using the novel methods for mechanical systems and biomedical systems, the paper "Automatic Condition Monitoring of Industrial Rolling-Element Bearings Using Motor's Vibration and Current Analysis" by Z. Yang presented an automatic condition monitoring system using motor vibration and current analysis to evaluate the work conditions of rollingelement bearings. In the paper "Application of $T^{2}$ Control Charts and Hidden Markov Models in Condition-Based Maintenance at Thermoelectric Power Plants" by E. Kisić et al., the condition-based maintenance systems using control charts and hidden Markov models were developed. In the paper "Determining Effects of Wagon Mass and Vehicle Velocity on Vertical Vibrations of a Rail Vehicle Moving with a Constant Acceleration on a Bridge Using Experimental and Numerical Methods" by C. Mizrak and I. Esen, the wagon mass and vehicle velocity indemnification system was developed. In the paper "Modeling and Simulation of ProcessMachine Interaction in Grinding of Cemented Carbide Indexable Inserts" by W. Feng et al., the grinding monitoring system was developed. In the paper "Real Time Cardan Shaft State Estimation of High-Speed Train Based on Ensemble Empirical Mode Decomposition" by C. Yi et al., the real time cardan shaft state monitoring system using ensemble 
empirical mode decomposition was developed. In the paper "Research on High-Frequency Combination Coding-Based SSVEP-BCIs and Its Signal Processing Algorithms" by F. Zhang et al., the evoked potential monitoring system was developed to monitor the electroencephalogram.

For the lightweight investigation using vibration signals for vehicle engineering, the paper "Lightweight Investigation of Extended-Range Electric Vehicle Based on Collision Failure Using Numerical Simulation" by J. Long et al. proposed a collision failure-based lightweight method to optimize the natural frequency and the corresponding vibration response of the extended-range electric vehicle.

For the numerical simulation of earthquake waveforms to the condition monitoring of civil engineering structures, the paper "Bizarre Waveforms in Strong Motion Records" by B. Zhou et al. investigated the abnormal waveform and found that it directly affects the characteristics of time history and frequency spectrum. The paper "The Influence of Pier Stiffness Ratio on the Failure Modes of Masonry Structures" by Y. Wang et al. investigated the influence of pier stiffness ratio on the failure mode of infrastructure when it afforded a heavy earthquake.

For the numerical simulation of structures, the paper "Free Vibration Analysis for Cracked FGM Beams by Means of a Continuous Beam Model” by E. C. Yang et al. proposed a numerical simulation method using continues beam model to analyze the functional graded materials (FGMs) beam with cracks. The paper "Identification of Crack Location in Beam Structures Using Wavelet Transform and Fractal Dimension" by Y.-Y. Jiang et al. presented wavelet-based numerical method and fractional dimension method to detect crack locations. The paper "Research on the Band Gap Characteristics of Two-Dimensional Phononic Crystals Microcavity with Local Resonant Structure" by M. Liu et al. presented novel two-dimensional phononic crystals (PCs) through numerical simulation, which might be employed to detect damage in structures. The paper "Resonance Analysis of HighFrequency Electrohydraulic Exciter Controlled by 2D Valve" by G. Pan et al. presented a numerical simulation method using Fourier series extension to analyze the conditions of electrohydraulic exciter controlled by two-dimensional valve. The paper "Theoretical Analysis and Experimental Verification of Particle Damper-Based Energy Dissipation with Applications to Reduce Structural Vibration" by X. Wangqiang et al. presented a numerical simulation method using theoretical analysis to reduce structural vibration of dampers.

In summary, much effort should be made to connect the novelty methods and the fault/damage detection for the real world mechanical and civil structures.

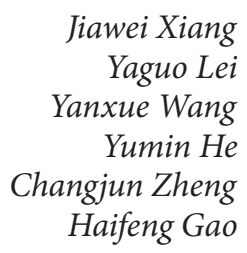

\section{References}

[1] L. J. Meng, J. W. Xiang, Y. X. Wang, Y. Y. Jiang, and H. F. Gao, "A hybrid fault diagnosis method using morphological filter-translation invariant wavelet and improved ensemble empirical mode decomposition," Mechanical Systems and Signal Processing, vol. 50-51, pp. 101-115, 2015.

[2] Y. Wang, R. Markert, J. Xiang, and W. Zheng, "Research on variational mode decomposition and its application in detecting rub-impact fault of the rotor system," Mechanical Systems and Signal Processing, vol. 60-61, pp. 243-251, 2015.

[3] Y. G. Lei, J. Lin, M. J. Zuo, and Z. J. He, "Condition monitoring and fault diagnosis of planetary gearboxes: a review," Measurement, vol. 48, no. 2, pp. 292-305, 2014.

[4] J. W. Xiang, Z. S. Jiang, and X. F. Chen, "A class of wavelet-based Rayleigh-Euler beam element for analyzing rotating shafts," Shock and Vibration, vol. 18, no. 3, pp. 447-458, 2011.

[5] J. Xiang and M. Liang, "A two-step approach to multi-damage detection for plate structures," Engineering Fracture Mechanics, vol. 91, pp. 73-86, 2012.

[6] J. W. Xiang, M. Liang, and Y. M. He, "Experimental investigation of frequency-based multi-damage detection for beams using support vector regression," Engineering Fracture Mechanics, vol. 131, pp. 257-268, 2014. 

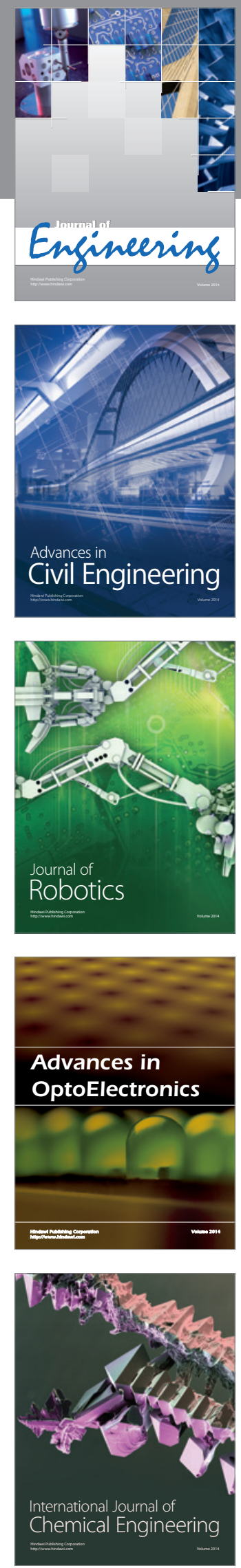

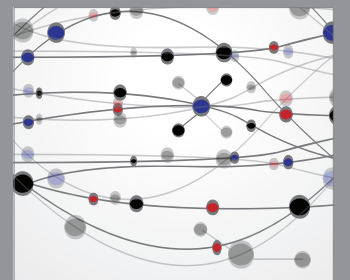

The Scientific World Journal
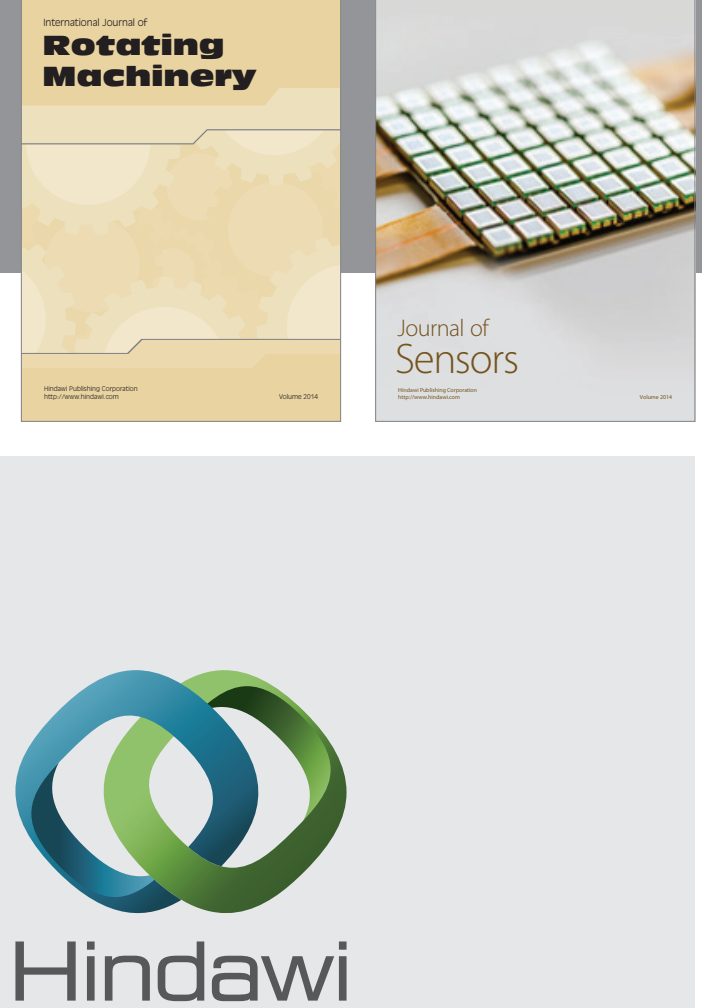

Submit your manuscripts at http://www.hindawi.com
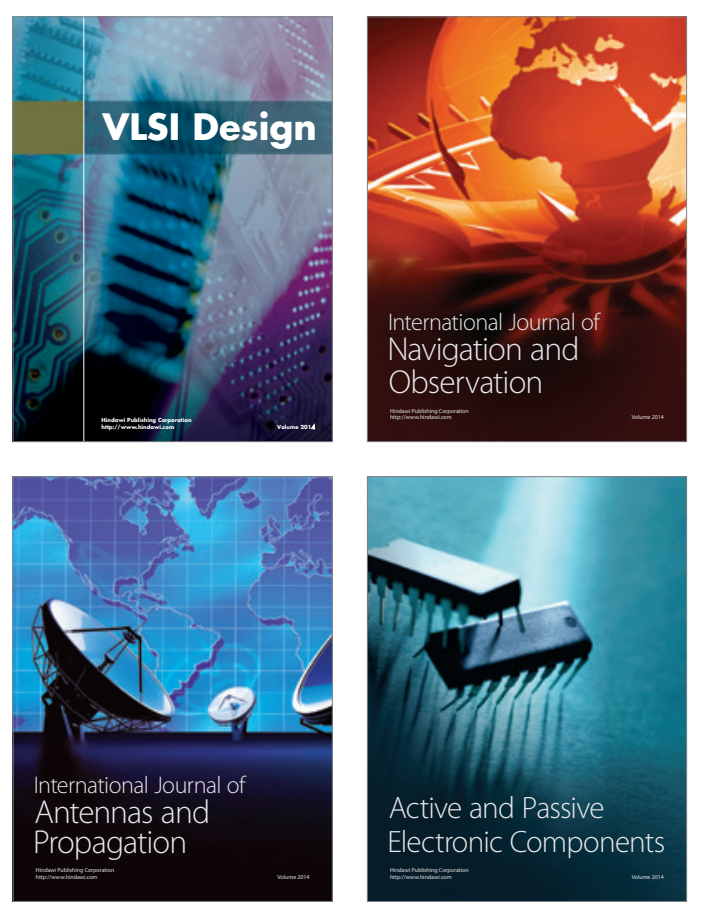
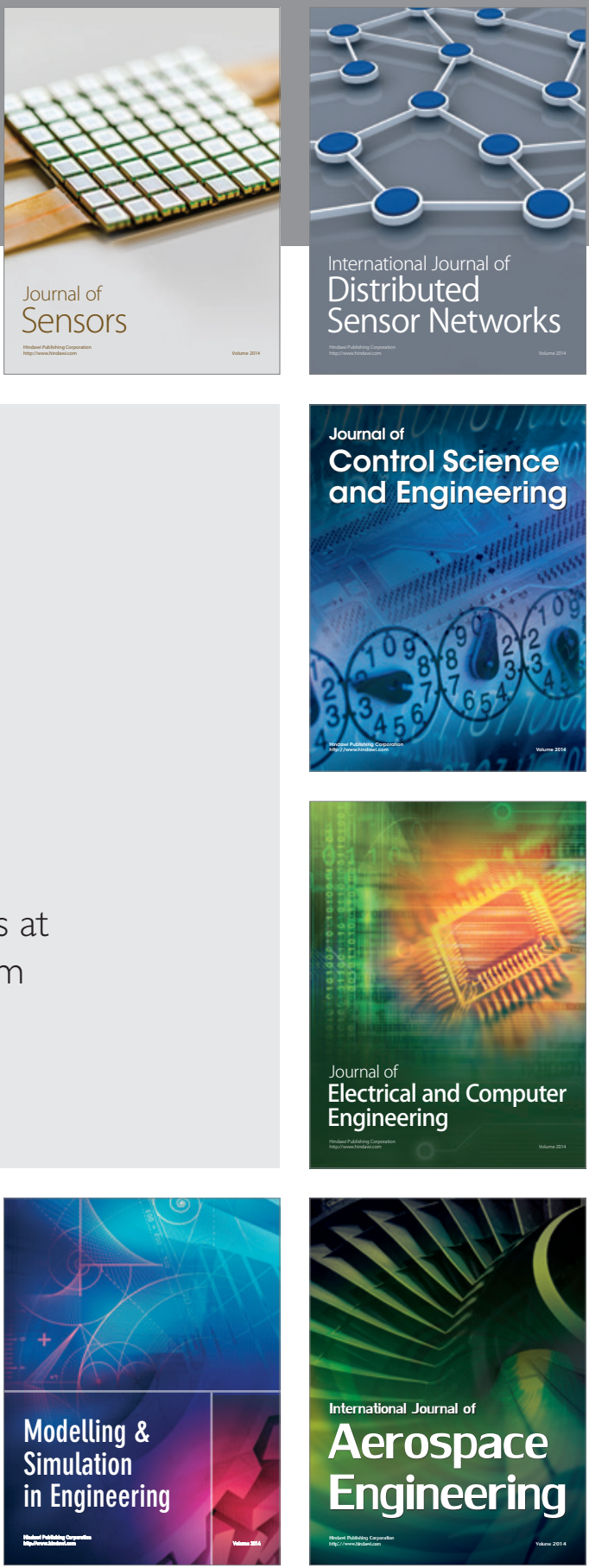

Journal of

Control Science

and Engineering
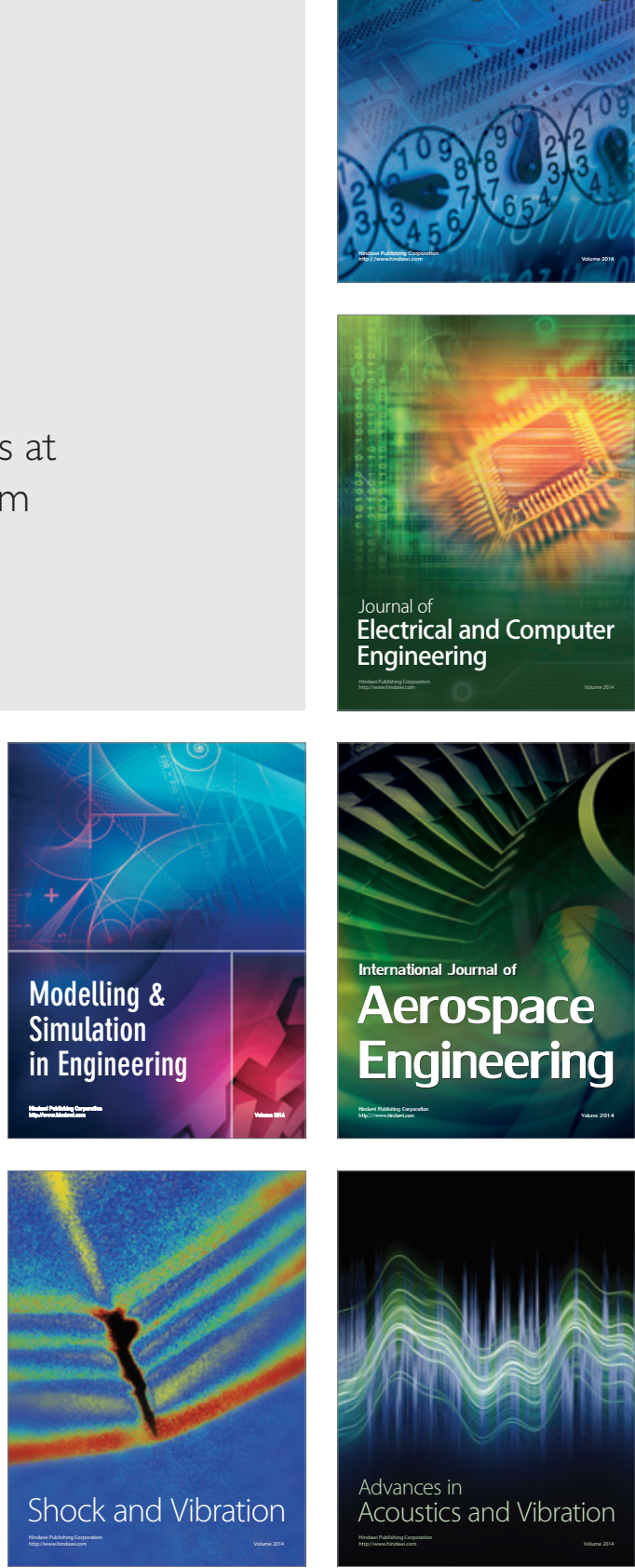